\title{
On spin independence in large Nc baryons
}

\section{Citation}

Carone, Chris, Howard Georgi, and Sam Osofsky. 1994. "On Spin Independence in Large Nc Baryons." Physics Letters B 322 (3): 227-32. https://doi.org/10.1016/0370-2693(94)91112-6.

\section{Permanent link}

http://nrs.harvard.edu/urn-3:HUL.InstRepos:41467471

\section{Terms of Use}

This article was downloaded from Harvard University's DASH repository, and is made available under the terms and conditions applicable to Other Posted Material, as set forth at http:// nrs.harvard.edu/urn-3:HUL.InstRepos:dash.current.terms-of-use\#LAA

\section{Share Your Story}

The Harvard community has made this article openly available.

Please share how this access benefits you. Submit a story.

Accessibility 
\#HUTP-93/A032

HEP-PH/9310365

$10 / 93$

\title{
On Spin Independence in Large $N_{c}$ Baryons $^{* \dagger}$
}

\author{
Chris Carone, Howard Georgi and Sam Osofsky \\ Lyman Laboratory of Physics \\ Harvard University \\ Cambridge, MA 02138
}

\begin{abstract}
We argue directly from Witten's analysis of large $N_{c}$ baryons that the structure of the $s$-wave low-spin baryon states in QCD becomes spin-independent as $N_{c} \rightarrow \infty$. This property leads to $S U(6)$-like behavior of static matrix elements, such as the axialvector current matrix elements recently studied by Dashen, Manohar and Jenkins. Our analysis suggests a method for applying large $N_{c}$ results for $N_{c}=3$, even though the baryon states for large $N_{c}$ are very different.
\end{abstract}

* Research supported in part by the National Science Foundation under Grant \#PHY-9218167.

${ }^{\dagger}$ Research supported in part by the Texas National Research Laboratory Commission, under Grant \#RGFY9206. 


\section{Introduction}

The classic paper by Witten on baryons in the large $N_{c}$ approximation shows that low-lying large $N_{c}$ baryons can be described by a Hartree wave-function with all (or almost all, for low-lying excited states) quarks in the same ground-state wave function, bound in a potential produced by all the other quarks. [1] One sentence in [1] hints that the spin structure of large $N_{c}$ baryonic bound states of light quarks may be an interesting thing to study. Witten notes that while spin-orbit coupling will seriously deform the high-spin baryons away from an $s$-wave ground state, the low-spin, ground state baryons may not be deformed. In this note, we attempt to make this notion precise. We will argue that spin-independence of large $N_{c}$ baryons emerges as an approximate symmetry of a rather unusual type. Spin dependent terms vanish as $N_{c} \rightarrow \infty$ when the baryon spin is held fixed. Thus the states of low spin in the baryon multiplet are spin-independent, while the states with spin of order $N_{c} / 2$ are seriously modified by spin-spin and spin-orbit interactions. We will then analyze the matrix elements of operators in these baryon states and show that for the ground states, the matrix elements have the structure suggested by spin-flavor symmetry arguments. Applied to the axial-vector current, this yields the results that Dashen and Manohar [2] and Jenkins [3] obtained by studying pion-baryon scattering. However, we see that the result is far more general, depending only large $N_{c}$, not on chiral symmetry.

One difficulty that arises in applying large $N_{c}$ ideas to baryons in our world is that baryon states for large $N_{c}$ look nothing like the baryons for $N_{c}=3$. It is purely coincidental that for only two flavors of quarks, the low lying states for any odd $N_{c}>3$ have the same quantum numbers as the states for $N_{c}=3$. For more than two flavors, the quantum numbers of the low-lying states look entirely different. It is thus important to extract results from a large $N_{c}$ analysis in a form that can be unambiguously applied to $N_{c}=3$. It seems clear that you should not directly compute properties of the large $N_{c}$ baryon states and then just take over the results to $N_{c}=3$. What we will suggest is to formulate the result as a sum over quark states, without regard to the value of $N_{c}$. This approach fits in nicely with the rest of our analysis.

We will work entirely within the Hartree picture of large $N_{c}$ baryons. However, to get started, we must know what spin states to consider. We begin by considering heavy quarks, as discussed explicitly by Witten. In this case, we know what the Hartree Hamiltonian looks like. The interactions are approximately spin independent simply because the quark masses are large and the baryons are nonrelativistic bound states. In this case, we know that the states are nonrelativistic states of 
$N_{c}$ quarks, each with two spins states and $f$ flavors states (for $f$ flavors), completely symmetric in spin, flavor and space variables. This is conveniently described in a $(2 f)^{N_{c}}$ dimensional tensor product space with independent spin indices and flavor indices for each of the quarks (labeled by $x=1$ to $N_{c}$ ). In the ground states, all the quarks will be in the same $s$-wave space wave function, and thus the states are completely symmetric in spin and flavor. The spin and flavor states are then described by a tower of (spin,flavor) representations of increasing spin as shown below (for odd $\left.N_{c}\right)$ :

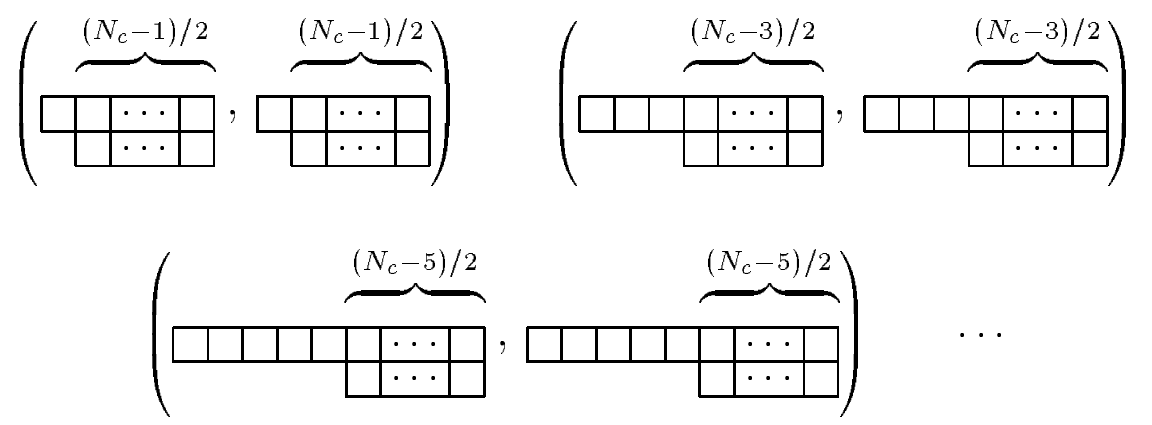

If the quarks are not heavy, we cannot in general ignore the spin dependent interactions. We do not know how to write down the Hartree potential. Nevertheless, we can plausibly argue that the low lying baryon states can be described in the same space, and by the same representations. For this to be the case, it is sufficient that no dramatic phase changes occur as we decrease the quark masses from large values, much greater than the QCD scale parameter $\Lambda$, down to small values, less than or of the order of $\Lambda$. This certainly appears to be true for $N_{c}=3$, where the baryon octet and decuplet bound states of the light $u, d$ and $s$ quarks correspond exactly to the states we would expect if the quarks were heavy. It would be quite bizarre if large $N_{c}$ worked very differently. ${ }^{1}$ In what follows, we will assume that this is correct and see what we can say about the states for large $N_{c}$.

\section{Energies}

We can write the energy of the baryon states in the Hartree language as follows:

$$
\sum_{n=1}^{N_{c}} H^{n}
$$

\footnotetext{
${ }^{1}$ This argument is independent of the Hartree approximation.
} 
where $n$ labels the number of quarks involved. It is convenient to think of this as a matrix in the $(2 f)^{N_{c}} \times(2 f)^{N_{c}}$ dimensional spin-flavor space of the baryon states. The $H^{n}$ are matrix functionals of the Hartree wave function, $\Phi(\vec{r})$, and it's adjoint, $\Phi(\vec{r})^{\dagger}$, where $\Phi$ is a $2 f \times 2 f$ matrix in spin and flavor space. The explicit expression involves a tensor product of $n \Phi \mathrm{s}$ and $n \Phi^{\dagger} \mathrm{s}$, one in each quark space,

$$
\begin{gathered}
H^{n}=\sum_{\substack{\left\{x_{1}, \cdots, x_{n}\right\} \\
\in\left\{1, \cdots, N_{c}\right\}}} \int d^{3} r_{x_{1}} \cdots d^{3} r_{x_{n}} \\
\Phi\left(\vec{r}_{x_{1}}\right)_{x_{1}}^{\dagger} \otimes \cdots \otimes \Phi\left(\vec{r}_{x_{n}}\right)_{x_{n}}^{\dagger} \tilde{h}^{n}\left(\vec{r}_{x_{1}}, \cdots, \vec{r}_{x_{n}}\right) \Phi\left(\vec{r}_{x_{1}}\right)_{x_{1}} \otimes \cdots \otimes \Phi\left(\vec{r}_{x_{n}}\right)_{x_{n}}
\end{gathered}
$$

where the $\tilde{h}^{n}$ s are $(2 f)^{N_{c}} \times(2 f)^{N_{c}}$ matrices acting on the spin and flavor space, $\left\{x_{1}, \cdots, x_{n}\right\}$ refers to a set of distinct quark lines $\left(x_{i} \neq x_{j}\right)$ and $\Phi\left(\vec{r}_{x}\right)_{x}$ is the Hartree wave function as a matrix acting in the space of the $x$ quark. Because each term in the sum in (3) describes an interaction that involves only the $n$ quarks in the set $\left\{x_{1}, \cdots, x_{n}\right\}$, the matrix is nontrivial only in a $(2 f)^{n} \times(2 f)^{n}$ subspace (a different one for each term in the sum). The matrix is the identity on the quark variables that are not in the set $\left\{x_{1}, \cdots, x_{n}\right\}$. In this note, we will discuss the limit in which the $f$ light quarks are degenerate, in which case there is an $S U(f)$ flavor symmetry, and the $\tilde{h}^{n}$ s are trivial in flavor space for all of the quarks, simply the product of identity matrices in each of the $f$ dimensional flavor spaces of the individual quark lines. We will return to the question of $S U(f)$ breaking in a future publication.

The trick that allows us to demonstrate the spin independence of the low-spin states is to break up the energy according to the transformation properties of the $\tilde{h}^{n}$ under the spin and orbital angular momentum generators on the various quark lines in the frame of reference in which the baryon is at rest. In particular, we will take as our "zeroth-order" term in the energy, the sum of the pieces of $\tilde{h}^{n}$ that are singlets under spin and orbital rotations, so that

$$
\tilde{h}^{n}\left(\vec{r}_{x_{1}}, \cdots, \vec{r}_{x_{n}}\right)=h_{0}^{n}\left(r_{x_{1}}, \cdots, r_{x_{n}}\right)+\Delta \tilde{h}^{n}\left(\vec{r}_{x_{1}}, \cdots, \vec{r}_{x_{n}}\right)
$$

and

$$
\begin{gathered}
H_{0}=\sum_{n=1}^{N_{c}} \sum_{\substack{\left\{x_{1}, \cdots, x_{n}\right\} \\
\in\left\{1, \cdots, N_{c}\right\}}} \int d^{3} r_{x_{1}} \cdots d^{3} r_{x_{n}} \\
\Phi\left(\vec{r}_{x_{1}}\right)_{x_{1}}^{\dagger} \otimes \cdots \otimes \Phi\left(\vec{r}_{x_{n}}\right)_{x_{n}}^{\dagger} h_{0}^{n}\left(r_{x_{1}}, \cdots, r_{x_{n}}\right) \Phi\left(\vec{r}_{x_{1}}\right)_{x_{1}} \otimes \cdots \otimes \Phi\left(\vec{r}_{x_{n}}\right)_{x_{n}}
\end{gathered}
$$


where the $h_{0}$ s are $c$-number functions (if you prefer, multiplied by the identity matrix in the full space) of the magnitudes of the coordinates, $r_{x} \equiv\left|\vec{r}_{x}\right|$. The remainder, $\Delta \tilde{h}^{n}\left(\vec{r}_{x_{1}}, \cdots, \vec{r}_{x_{n}}\right)$, transforms nontrivially under the spin and/or orbital angular momentum on at least one of the quark lines.

The plan now is to imagine using $H_{0}$ to determine the Hartree potential, and then treat the $\Delta \tilde{h}^{n}\left(\vec{r}_{x_{1}}, \cdots, \vec{r}_{x_{n}}\right)$ as a perturbation. Note that the $h_{0}^{n}$ for $n>1$ contribute in large $N_{c}$ even though they are of order $1 / N_{c}^{n-1}$, because combinatoric factors from the sum in (3) cancel the $N_{c}$ dependence of the individual graphs. [1] While we cannot determine the Hartree potential for light quarks explicitly, even in this approximation, it is clear that resulting ground-state Hartree wave functions will be spin independent and functions only of $r_{x}$, so that the matrix functions, $\Phi\left(\vec{r}_{x}\right)$ become $c$-numbers:

$$
\Phi\left(\vec{r}_{x}\right) \rightarrow \phi\left(r_{x}\right)
$$

In this approximation, all the spin states in the ground-state baryon multiplet are degenerate and have the same space wave function. We can now put the $s$-wave, spin independent Hartree wave functions back into the full expression for the energy and ask what is the effect if the spin dependent terms. Roughly speaking, we will find that while the terms in $H_{0}$ involving two or more quarks add coherently to give a large effect, the spin dependent terms add incoherently for the low spin states. This is why the spin dependent terms have a small effect.

\section{Splittings}

Let us now consider in detail the splittings introduced by $\Delta \tilde{h}^{n}\left(\vec{r}_{x_{1}}, \cdots, \vec{r}_{x_{n}}\right)$. We will need three simple facts:

1. $\Delta \tilde{h}^{n}\left(\vec{r}_{x_{1}}, \cdots, \vec{r}_{x_{n}}\right)$ is of order $1 / N_{c}^{(n-1)}$;

2. Terms transforming nontrivially under orbital angular momentum on any quark line vanish when integrated over space in $(3)$;

3. The spin matrix structure on each quark line (labeled by $x$ ) can always be written in the form $a+\vec{b} \cdot \vec{\sigma}_{x}$, where $\vec{\sigma}_{x}$ are the Pauli matrices acting on the quark line.

Using these facts, the integrations in (3) can be formally done, and the results replaced by unknown constants, so that energy has the following form (still a matrix in the $(2 f)^{N_{c}} \times(2 f)^{N_{c}}$ spin-flavor 
space):

$$
N_{c} E_{0}+\sum_{n=1}^{N_{c}} \frac{1}{N_{c}^{n-1}} \sum_{\substack{\left\{x_{1}, \cdots, x_{n}\right\} \\ \in\left\{1, \cdots, N_{c}\right\}}} \sum_{\substack{a_{1} \\ \text {, }, \cdots, a_{x_{n}} \\=0}}^{3} \sigma_{x_{1}}^{a_{x_{1}}} \cdots \sigma_{x_{n}}^{a_{x_{n}}} k^{a_{x_{1}} \cdots a_{x_{n}}},
$$

where we have defined $\sigma_{x}^{0} \equiv I$. Note that the tensor, $k$ is completely symmetric.

The next step is note that all the terms proportional to $\sigma_{x}^{0}$ on any quark line, $x$, are actually irrelevant. For these terms, we can do the sum over $x$ explicitly, picking up a factor of order $N_{c}$. The result looks just like the term in (7) with one less quark line $(n \rightarrow n-1)$. Thus if we eliminate all the $\sigma_{x}^{0}$ s, it simply changes the values of the unknown parameters in $(7)$, and we can write the energy as

$$
N_{c} E_{0}+\sum_{n=2}^{N_{c}} \frac{1}{N_{c}^{n-1}} \sum_{\substack{\left\{x_{1}, \cdots, x_{n}\right\} \\ \in\left\{1, \cdots, N_{c}\right\}}} \sum_{\substack{a_{1} \\ \text {, } \\=1}}^{3} \sigma_{x_{n}}^{a_{x_{1}}} \cdots \sigma_{x_{n}}^{a_{x_{n}}} k^{a_{x_{1}} \cdots a_{x_{n}}} .
$$

Note that the $n=1$ term in the sum has disappeared because of rotation invariance. There is no way to build a spin-dependent term with only one $\vec{\sigma}$ in the $s$-wave ground state.

Finally, consider what happens if instead of summing over the sets $\left\{x_{1}, \cdots, x_{n}\right\}$, we sum independently over the individual quark lines. This introduces combinatoric factors, but for small $n$, they are of order 1 as $N_{c} \rightarrow \infty$ and can be absorbed into the unknown coefficients. We also make errors by including contributions when two or more quarks lines in the sum are the same, but these are always down by powers of $N_{c}$ compared to contributions we keep because they involve fewer sums over quark lines. Thus we can write the energy as

$$
\begin{gathered}
N_{c} E_{0}+\sum_{n=2}^{N_{c}} \frac{1}{N_{c}^{n-1}} \sum_{\substack{x_{1}, \cdots, x_{n} \\
=1}}^{N_{c}} \sum_{\substack{a_{x_{1}}, \cdots, a_{x_{n}} \\
=1}}^{3} \sigma_{x_{1}}^{a_{x_{1}} \cdots \sigma_{x_{n}}^{a_{x_{n}}} k^{a_{x_{1}} \cdots a_{x_{n}}}} \\
=N_{c} E_{0}+N_{c} \sum_{n=2}^{N_{c}} \sum_{\substack{a_{1}, \cdots, a_{n} \\
=1}}^{3} \frac{2}{N_{c}} S_{1}^{a_{1}} \cdots \frac{2}{N_{c}} S_{n}^{a_{n}} k^{a_{1} \cdots a_{n}}=N_{c} F\left(S^{2} / N_{c}^{2}\right),
\end{gathered}
$$

where $\vec{S}$ is the total spin of the baryon. Though we derived this result thinking about first order perturbation theory in $\Delta \tilde{h}^{n}\left(\vec{r}_{x_{1}}, \cdots, \vec{r}_{x_{n}}\right)$, it is actually completely general - it is clear that the counting of powers of $N_{c}$ in higher order terms goes exactly the same way.

(9) has the property promised in the introduction. The spin dependent corrections are small (order $1 / N_{c}$ - two factors of $N_{c}$ smaller than the total energy) for fixed spin as $N_{c} \rightarrow \infty$. However, near the top of the ground-state multiplet where $\vec{S}=\mathcal{O}\left(N_{c} / 2\right)$, the corrections are as large as the zeroth order term. For the top of the multiplet, the perturbation theory breaks down completely and the Hartree wave function for the high spin states is not simply related to $\phi(r)$. 


\section{Matrix Elements}

All of the above, we believe, is well known to workers in the field, although we have not seen it expressed in this way in the literature. The advantage of the systematic approach described above is that we can now apply the same ideas to discuss matrix elements of operators between baryon states. The results will be similar to those for the baryon energies. We will find large $N_{c}$ predictions for the matrix elements that are corrected by terms that are smaller by a factor of order $S^{2} / N_{c}^{2}$. Thus the predictions should be reliable at the bottom of the ground state baryon multiplet.

Consider first the matrix elements of two-quark operators of the form

$$
\bar{\psi} \Gamma \psi
$$

where $\Gamma$ is a product of a flavor matrix $\Lambda$ times some $\gamma$ matrix. In a large $N_{c}$ baryon state, the matrix element will involve the same flavor matrix, $\Lambda$, and a matrix, $\kappa$, in spin space that is either 1 or $\vec{\sigma}$ depending on whether the matrix element is a scalar or a vector under rotations. There may also be orbital contributions to the spin structure of the operator, but these are irrelevant to matrix elements in the low-spin ground states because the expectation values are computed in the $s$-wave Hartree wave functions. Thus the form of the operator on any single quark line, $x$, is proportional to $\Lambda_{x} \kappa_{x}$, where $\Lambda_{x}$ and $\kappa_{x}$ are just the matrices $\Lambda$ and $\kappa$ acting on the flavor and spin spaces of the $x$ quark.

Arguments precisely analogous to those that we used to discuss the energies then imply that the leading order contribution to the matrix element of the operator (which like the energy, we will express as a $(2 f)^{N_{c}} \times(2 f)^{N_{c}}$ matrix in the spin-flavor space) has the form:

$$
\sum_{x=1}^{N_{c}}\left(a \Lambda_{x} \kappa_{x}+b \sigma_{x}^{a} \Lambda_{x} \kappa_{x} \sigma_{x}^{a}\right),
$$

where $a$ and $b$ are constants. The second term in (11) arises because there are effects that are leading order in $N_{c}$ from diagrams of the form shown in Fig. 1, where the shaded region represents some planar collection of gluon lines. This term does not have a dramatic effect on the form of the matrix element, but it allows for the possibility that vector and scalar operators are renormalized differently. Except for this unknown difference in normalization of vector and scalar operators, (11) is the standard result of $S U(2 f)$ symmetry arguments. Note also that the constants $a$ and $b$ will depend on the details of the $\gamma$ matrix structure in (10). For example, the space component of the axial vector current, with $\Gamma=\Lambda \vec{\gamma}$ will yield different values of $a$ and $b$ than the operator with 
$\Gamma=\Lambda \sigma^{a b}$, even though both transform like vectors. As noted by Dashen and Manohar [2], the matrix elements (11) can be of order $N_{c}$ because of the sum over quarks.

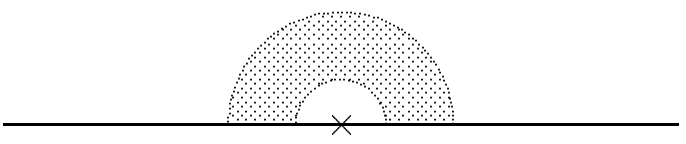

Figure 1: General diagram contributing to renormalization of two-quark operators.

Our result, (11), contains the results of Dashen and Manohar [2] and Jenkins [3] for the isovector axial vector current, but it is more general. For example, it predicts similar $S U(6)$ relations for the isoscalar axial vector current as well.

\section{Four-quark operators}

Next consider matrix elements of four-quark operators, such as

$$
\bar{\psi} \Gamma^{1} \psi \bar{\psi} \Gamma^{2} \psi
$$

As above, the matrix element in large $N_{c}$ baryons will involve the substitution

$$
\Gamma^{1} \rightarrow \Lambda^{1} \kappa^{1}, \quad \Gamma^{2} \rightarrow \Lambda^{2} \kappa^{2}
$$

where the $\kappa^{j}$ s are $\sigma^{j}$ s or identity matrices in spin space depending on whether $\Gamma^{j}$ is a vector or scalar under rotations.

Now by the usual argument, the leading large $N_{c}$ prediction for the matrix elements in the ground-state baryon states is

$$
\begin{gathered}
\sum_{\substack{x_{1}, x_{2} \\
=1}}^{N_{c}}\left(a_{1} \Lambda_{x_{1}}^{1} \kappa_{x_{1}}^{1} \Lambda_{x_{2}}^{2} \kappa_{x_{2}}^{2}+a_{2} \sigma_{x_{1}}^{a} \Lambda_{x_{1}}^{1} \kappa_{x_{1}}^{1} \sigma_{x_{1}}^{a} \Lambda_{x_{2}}^{2} \kappa_{x_{2}}^{2}\right. \\
\left.+a_{3} \Lambda_{x_{1}}^{1} \kappa_{x_{1}}^{1} \sigma_{x_{2}}^{a} \Lambda_{x_{2}}^{2} \kappa_{x_{2}}^{2} \sigma_{x_{2}}^{a}+a_{4} \sigma_{x_{1}}^{a} \Lambda_{x_{1}}^{1} \kappa_{x_{1}}^{1} \sigma_{x_{1}}^{a} \sigma_{x_{2}}^{a} \Lambda_{x_{2}}^{2} \kappa_{x_{2}}^{2} \sigma_{x_{2}}^{a}\right)
\end{gathered}
$$

which, because of the double sum, can grow like $N_{c}^{2}$ as $N_{c} \rightarrow \infty$.

It should be clear to the reader how similar predictions can be obtained for matrix elements of operators with more quark fields. 


\section{Comments}

It is worth restating the warning in the introduction about the application of these predictions to $N_{c}=3$. In our view, the only sensible way to proceed is to take the result (11) or (14) and apply it for $N_{c}=3$, because real large $N_{c}$ baryons simply do not look anything like $N_{c}=3$ baryons. Unfortunately, this is not always done. For example, calculations in Skyrmion models [4] are not consistent with this view. A particularly obvious problem with Skyrmion calculation is that they yield non-zero proton matrix-elements of $\bar{s} s$ operators. It is clear that these matrix elements are non-zero for large $N_{c}$ for precisely the same reason that the large $N_{c}$ baryons and the $N_{c}=3$ baryons have very different quantum numbers. The strange quarks in the large $N_{c}$ baryon are not part of the sea. They are valence quarks! The $\bar{s} s$ operators have nonzero matrix elements in large $N_{c}$ baryons simply because the large $N_{c}$ baryons have the wrong valence structure. Our prediction for matrix elements of $\bar{s} s$ operators, based on (11) or (14) or their generalizations, is zero to leading order in $N_{c}$, as you should expect from the absence of quark loops in leading order large $N_{c}$ calculations.

In this paper, we have set up a formalism that is useful for exploiting the approximate spin independence of low spin baryon states in a systematic way. In a future publication, we will give some examples of applications of (11) and (14) and discuss the effects of $S U(f)$ flavor symmetry breaking. We will also give explicit examples of $1 / N_{c}$ corrections and show how to apply these arguments to excited baryon states.

\section{Acknowledgements}

HG is grateful for useful conversations with Roger Dashen, Bob Jaffe, David Kaplan, Aneesh Manohar and Ann Nelson.

\section{References}

[1] E. Witten, Nucl. Phys. B160 (1979) 57.

[2] R. Dashen and A.V. Manohar, Phys. Lett. B315 (1993) 425, and 438.

[3] E. Jenkins, Phys. Lett. B315 (1993) 431, 441 and 447.

[4] See S.J. Brodsky, J. Ellis and M. Karliner, Phys. Lett. B206 (1988) 309, and references therein. 